\title{
Charge Collection and SEU from Angled Ion Strikes
}

\author{
P. E. Dodd, M. R. Shaneyfelt, and F. W. Sexton \\ Sandia National Laboratories \\ Albuquerque, New Mexico 87185-1083
}

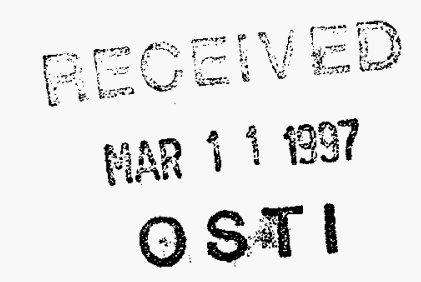

Phone: (505) 844-1447

FAX: (505) 845-8316

E-mail: pedodd@sandia.gov

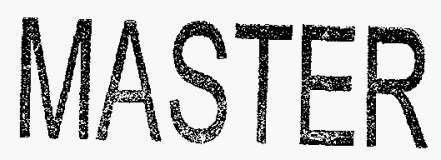

Abstract- Charge collection and SEU from angled ion strikes are studied using threedimensional simulation. The inverse cosine scaling law for angled strikes is shown to be of limited usefulness. Impacts on SEU test methodology are discussed.

This work was supported by the United States Department of Energy under contract DEAC04-94AL85000. Sandia is a multiprogram laboratory operated by Sandia Corporation, a Lockheed Martin Company, for the United States Department of Energy. 


\section{DISCLAIMER}

This report was prepared as an account of work sponsored by an agency of the United States Government. Neither the United States Government nor any agency thereof, nor any of their employees, make any warranty, express or implied, or assumes any legal liability or responsibility for the accuracy, completeness, or usefulness of any information, apparatus, product, or process disclosed, or represents that its use would not infringe privately owned rights. Reference herein to any specific commercial product, process, or service by trade name, trademark, manufacturer, or otherwise does not necessarily constitute or imply its endorsement, recommendation, or favoring by the United States Government or any agency thereof. The views and opinions of authors expressed herein do not necessarily state or reflect those of the United States Government or any agency thereof. 


\section{DISCLAMIER}

Portions of this document may be illegible in electronic image products. Images are produced from the best available original document. 


\title{
Charge Collection and SEU from Angled Ion Strikes
}

\author{
P. E. Dodd, M. R. Shaneyfelt, and F. W. Sexton \\ Sandia National Laboratories, Albuquerque, New Mexico 87185-1083
}

\section{Introduction}

Single event upsets (SEUs) are caused in semiconductor microcircuits when charge is deposited in a sensitive volume of the circuit by an incident energetic particle. Collection of this charge causes a loss of information stored at the struck circuit node. Sensitive regions of a microcircuit typically consist of reverse-biased junctions which efficiently collect deposited charge through the influence of drift fields.

During laboratory SEU testing, angled ion strikes are often used to conveniently mimic normally incident particles of higher linear energy transfer (LET) [1,2]. This practice is based on ion pathlengths through a thin rectangular parallelepiped (RPP) sensitive volume. Specifically, we assume that an angled strike deposits $1 / \cos \theta$ more charge in the sensitive volume, which in turn is assumed to lead to $1 / \cos \theta$ more charge collection at the sensitive node, and an increase in the particle's effective LET to $1 / \cos \theta$ higher than at normal incidence. This concept is mathematically stated by the familiar inverse cosine law:

$$
L E T_{\text {eff }}=L E T_{0} / \cos \theta,
$$

where $L E T_{\text {eff }}$ is the particle's effective LET, $L E T_{0}$ is the particle's true LET, and $\theta$ is the particle's entry angle with respect to normal incidence. Thus, for a given particle and energy, the effective LET of the particle is doubled at an angle of incidence of 60 degrees. In performing such experiments it is also sometimes necessary to independently correct the measured upset cross-section by the same $\cos \theta$ factor due to geometric considerations [1,3]; this separate issue will not be discussed in this summary.

Although the concept of effective LET has received widespread use, it suffers from inherent failings $[4,5]$. Of concern here are the breakdown of the "thin" RPP assumption for small-geometry devices (i.e., the RPP becomes box-shaped rather than slab-shaped [6]), and even more importantly, the inverse cosine law's neglect of charge collection by diffusion. Both of these problems can invalidate the assumption of direct correspondence between charge deposition in the sensitive volume and charge collection at the sensitive node. The breakdown of the thinness assumption can invalidate the $1 / \cos \theta$ increase of charge deposition in the RPP. This can be handled by simple geometric corrections to the inverse cosine law (e.g., accounting for particles that exit through the side of the junction rather than the bottom). Failure to account for diffusive charge collection is a more basic weakness of the RPP model for sensitive volumes. Corrections have been proposed, but they inherently result in non-boxshaped sensitive volumes [4].

Another assumption implicit to the use of Eq. (1) is the supposed direct proportionality between charge collection and effective LET. It has been shown that, for some circuits, there is no direct (or even monotonic) relation between total collected charge and upset threshold [7]. In static circuits such as SRAMs, the time over which charge is collected is as important as the amount of charge collected. If an angled strike exhibits a different charge collection time profile than an equivalent normally-incident strike, Eq. (1) is expected to be invalid for static circuits.

Experimental treatments of angled-strike charge collection have been performed $[3,8]$. Results for largearea diodes have shown relatively good agreement between angled strike charge collection and the inverse cosine law for effective LET [8]. More recent experiments have shown clear deviations from $1 / \cos \theta$ behavior, including rotation orientation dependence for rectangular devices with one axis longer than the other [3]. Simulation data are limited [9-11], but have shown that for inside-the-well transistors, or outside-the-well transistors with very small gate lengths, charge multiplication can occur for ions angled toward the source, a clear violation of the inverse cosine law $[9,11]$. Even simple large-area diode charge-collection simulations have shown deviations from $1 / \cos \theta$ behavior.

In this summary, we examine diode charge collection characteristics and SRAM upset thresholds as a function of ion angle of incidence, using threedimensional mixed-level simulation. Diode charge collection results provide basic physical insight and can be directly applied to DRAM structures. 


\section{Diode Charge Collection}

\section{Simulation Method}

In this work we have used the three-dimensional device simulator DAVINCI [12], which solves Poisson's equation and the current continuity equations using finite-element techniques. Physical models used in the simulation included carrier concentrationdependent minority carrier lifetimes, Auger recombination, and mobility models which included doping, electric field, and carrier-carrier scattering dependence. The technology modeled was based on Sandia's CMOS6 p-epitaxial-substrate process [13].

We have simulated charge collection in both $n-$ drain/p-epi (outside-the-well) and p-drain/n-well $/ \mathrm{p}$ epi (inside-the-well) reverse-biased junctions. A diagram of the simulation setup is shown in Figure 1 for inside-the-well simulations. The drain areas are $2 \mu \mathrm{m}$ square, the $n$-well area is $4 \mu \mathrm{m}$ square, and the epitaxial layer thickness is about $2.5 \mu \mathrm{m}$. The simulation volume extended well beyond (10s of microns) the ion strike in all cases. A $100-\mathrm{MeV} \mathrm{Fe}$ strike to the center of the junction was used to study the variation of charge collection with angle of incidence.

\section{Outside-the-Well Diode Results}

The results of outside-the-well angled strike charge collection simulations are shown in Figure 2. The ratio of total charge collection at a given angle divided by the normal-incidence charge collection is plotted vs. angle of incidence. Simulations were performed for two minority carrier lifetimes, $\tau=1 \mu \mathrm{s}$ and $\tau=0.1 \mu \mathrm{s}$. By comparison to the $1 / \cos \theta$ curve, it is clear that the collected charge does not increase as quickly as is predicted by inverse cosine law. That the curve for $\tau=$ $0.1 \mu$ s increases more rapidly than the curve for $\tau=1$ $\mu$ indicates that charge being collected by diffusion is at least partly responsible for the cosine law discrepancy.

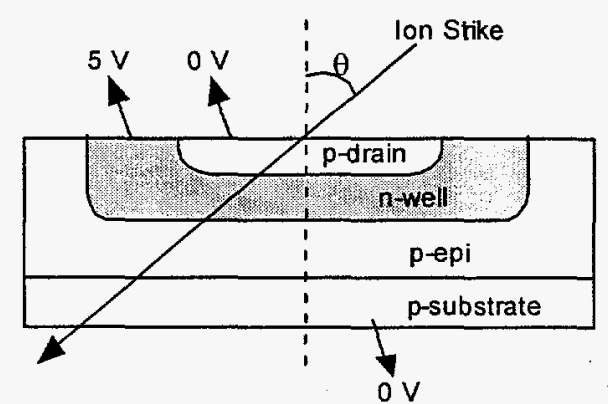

Figure 1. Illustration of an angled ion strike to the center of an inside-the-well diode.

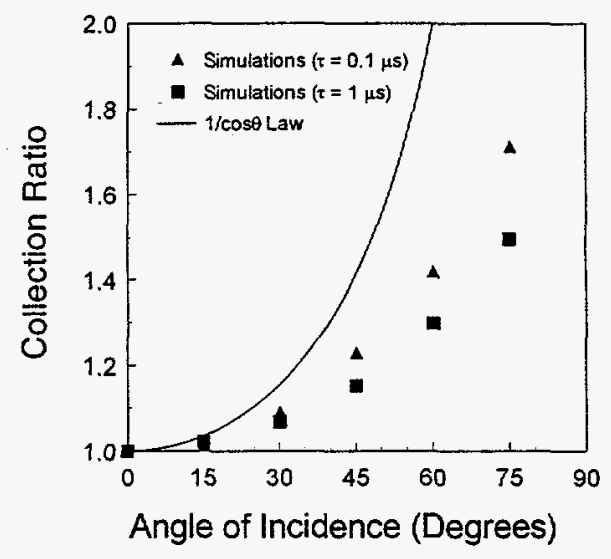

Figure 2. Simulated outside-the-well diode charge collection from angled strikes normalized to zero-angle collection.

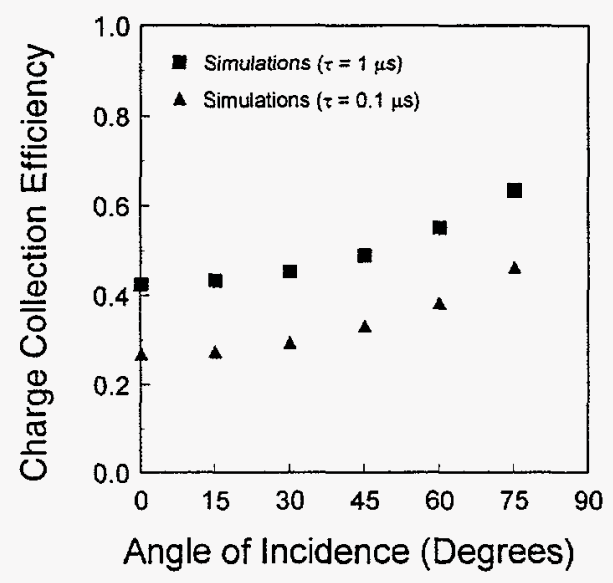

Figure 3. Simulated outside-the-well diode charge collection efficiency as a function of ion angle.

If we examine the absolute charge collection efficiency as a function of angle (Figure 3), we find further evidence of diffusive collection. For ion strikes to outside-the-well diodes on an epitaxial substrate, a typical assumed collection depth would be the epitaxial layer thickness, with a funnel depth extending down to the substrate for angled strikes [14]. In Figure 3 for the larger-lifetime simulation (solid squares), we find a charge collection efficiency at normal incidence of about $42 \%$, implying an equivalent charge collection depth of over $8 \mu \mathrm{m}$ (more than $5 \mu \mathrm{m}$ below the epi). As expected, lower collection efficiencies are computed in the lower-lifetime simulations. Considerable charge collection depths in heavily-doped epitaxial substrates have recently been experimentally measured, further reaffirming the importance of diffusive collection from deep within epitaxial structures [15].

Charge collection by drift can be determined from simulations by examining the charge collection time. profile, where for epitaxial structures a clear inflection 
is seen as collection changes from drift-dominated to diffusion-dominated [16]. Counting only this drift charge, simulated collection ratios for both lifetimes are shown in Figure 4. We see that now the results for both lifetimes are equivalent (as expected), but still below the standard inverse cosine law. From the simulated drift charge-collection efficiency at normal incidence we find a drift charge-collection depth of $4 \mu \mathrm{m}$ beneath the drain, or an apparent funnel depth of $1.8 \mu \mathrm{m}$ below the epi layer. If we assume that the funnel length in the substrate is invariant with angle [4], we can construct a corrected geometric model for drift charge collection which is seen to agree more closely with the simulation results. Details of this simple analytical model will be provided in the full paper. Apparently the breakdown of the inverse cosine law for outside-the-well diode strikes is due to a combination of diffusive collection and angle-invariant funneling. Implications of these results for bulk and thick epi technologies will be discussed in the full paper.

\section{Inside-the-Well Diode Results}

For ion strikes to inside-the-well diodes, charge deposited in the well can be collected at either the reverse-biased drain/well junction or the reversebiased well/epi junction (see Figure 1). A reasonable first-order estimate might be a sensitive volume defined by the midpoints between the drain and epi boundaries $^{\dagger}$. For the junction and well sizes and depths simulated here, it can easily be shown that the inverse cosine law will fail geometrically at around a 63 degree angle of incidence due to ions exiting the side rather

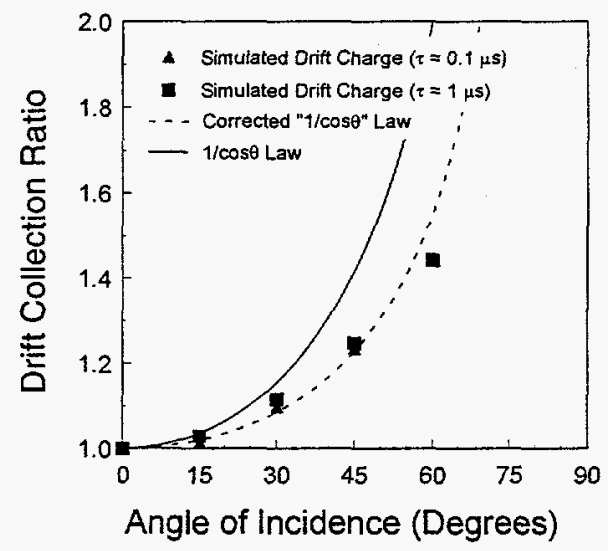

Figure 4. Simulated outside-the-well drift charge collection as a function of angle compared to geometric models.

\footnotetext{
+ This is not exactly correct, but serves to illustrate the nature of geometric corrections necessary for the $1 / \cos \theta$ law.
}

than the bottom of the sensitive volume, and that another charge collection regime exists above 73 degrees as particles exit the side of the struck junction itself. Another geometrically-based analytical model can be constructed to correct the $1 / \cos \theta$ law for insidethe-well strikes at large angles, and is shown in Figure 5 along with the original inverse cosine law and simulation results.

It is immediately apparent from Figure 5 that, for the geometries considered here, simple drift-based geometric models describe total charge collection for inside-the-well strikes better than for outside-the-well strikes. This should come as no great surprise, since diffusion and funneling play little role in inside-thewell diode charge collection for the bias conditions shown in Figure 1. We note, however, that if the top junction were biased at $5 \mathrm{~V}$ (mimicking the p-channel ON condition), we would expect to see shunting between the drain and substrate which might significantly alter the angular dependence of charge collection. Also, if we had simulated a full transistor rather than just the drain junction, we would expect to see enhanced drain collection with increased angle of incidence due to source barrier lowering [9]. In the full paper this subject will be explored in greater detail.

\section{SRAM Upset Thresholds}

\section{Simulation Method}

Simulation of upset thresholds in an SRAM requires the simultaneous solution of device and circuit node equations, or mixed-level simulation. In this work we have used DAVINCI, modeling the struck transistor in three dimensions and the rest of the

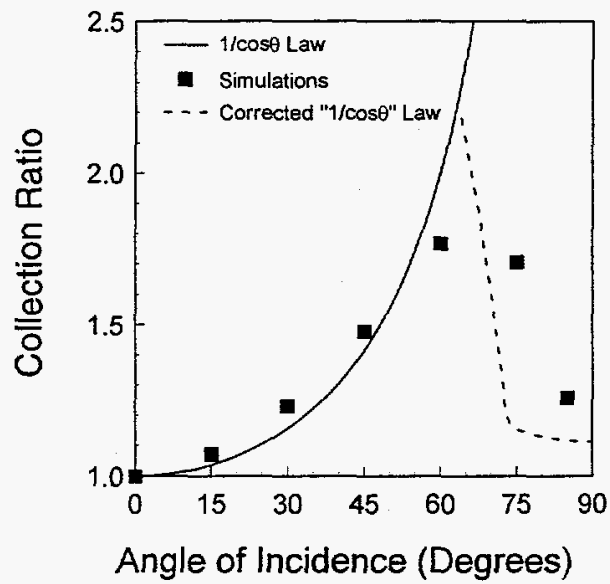

Figure 5. Simulated inside-the-well diode charge collection from angled strikes normalized to zero-angle collection. 
SRAM cell with circuit equations [7]. The modeled SRAM cell did not have feedback resistors. Finding the upset threshold is an iterative process, where we scale the LET of the $100-\mathrm{MeV} \mathrm{Fe}$ structure ion strike until we find the threshold which will just induce upset. For the SRAM simulations, all strikes were angled through the device centerline from the drain toward the source.

\section{Outside-the-Well Strikes}

Simulated upset thresholds are shown in Figure 6 for ion strikes to the center of the drain for outsidethe-well OFF transistors ( $\mathrm{n}$-channel in this $\mathrm{p}$-substrate technology). We have plotted the upset threshold LET normalized to the zero-angle result and $\cos \theta$. In other words, if the inverse cosine law held, the normalized threshold would be angle-invariant (straight line in Figure 6). Instead, we find that a higher than expected incident LET must be used for angled strikes to reach the upset threshold. At an angle of 60 degrees, an effective LET of 1.5 times higher than the zero-angle threshold normalized by $\cos \theta$ is required to produce an upset. The expected impact of such a result on SEU cross-section measurements will be discussed below. In the full paper we will present SRAM results for insidethe-well strikes and ions angled both towards and away from the source.

\section{Critical Charge and Angled Strikes}

Critical charge to upset is a convenient parameter used to characterize a circuit's upset sensitivity and to predict error rates. We define the critical charge as the minimum collected charge that will cause an upset. Critical charge is most useful for upset rate calculations if the critical charge for a sensitive volume does not

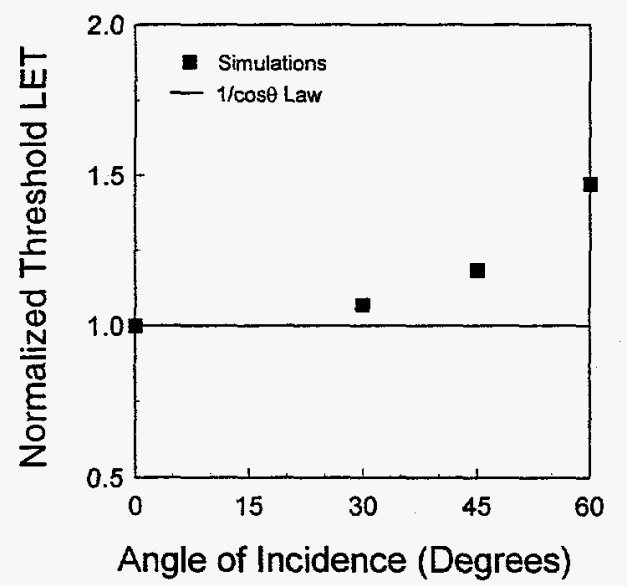

Figure 6. Normalized threshold LET for ion strikes to outsidethe-well transistors in an SRAM. Note the cosine law produces a constant normalized threshold LET. depend on angle of incidence. This allows charge deposition along sensitive volume chordlengths to be compared to a single-valued critical charge.

Using the simulations of Figure 6, we have computed critical charge as a function of angle for the modeled SRAM cell [7]. We find the critical charge to be about 55-60 fC for all angles of incidence, confirming the validity of a single-valued critical charge $v s$. angle for outside-the-well transistors in this technology.

\section{Conclusions}

In today's small-geometry devices, the assumptions inherent to the inverse cosine law are no longer uniformly applicable. The inverse cosine law works reasonably well for inside-the-well strikes, but fails when diffusive collection is important (e.g., outsidethe-well strikes). Models of diffusive charge collection have been developed $[8,17,18]$, and may prove to be useful for correcting SEU measurements taken with angled ions. The implications of inverse cosine law failure for SEU test procedures will be discussed in detail in the full paper.

\section{References}

[1] F. W. Sexton, 1992 IEEE NSREC Short Course, New Orleans, LA.

[2] S. Duzellier et al., IEEE Trans. Nucl. Sci., 43, p. 671, 1996.

[3] P. J. McNulty et al., IEEE Trans. Nucl. Sci., 39, p. 1622, 1992.

[4] L. W. Connell et al., IEEE Trans. Nucl. Sci., 42, p. 73, 1995.

[5] J. C. Pickel, IEEE Trans. Nucl. Sci., 43, p. 483, 1996.

[6] E. L. Petersen et al., IEEE Trans. Nucl. Sci., NS-30, p. 4533, 1983.

[7] P. E. Dodd et al., IEEE Trans. Nucl. Sci., 42, p. 1764, 1995.

[8] A. B. Campbell et al., IEEE Trans. Nucl. Sci., NS-30, p. 4486, 1983.

[9] R. L. Woodruff et al., IEEE Trans. Nucl. Sci., 40, p. 1795, 1993.

[10] H. Dussault et al., Proc. RADECS 93, p. 509, 1993.

[11] S. Velacheri et al., IEEE Trans. Nucl. Sci., 41, p. 2103, 1994.

[12] DAVINCI 3.2 User's Manual (Technology Modeling Associates, Inc., 1996).

[13] P. E. Dodd et al., IEEE Trans. Nucl. Sci., 43, p. 2797, 1996.

[14] K. W. Golke, IEEE Trans. Nucl. Sci., 40, p. 1910, 1993.

[15] H. Dussault et al., IEEE Trans. Nucl. Sci., 41, p. 2018, 1994.

[16] P. E. Dodd et al., IEEE Trans. Nucl. Sci., 41, p. 2005, 1994.

[17] S. Kirkpatrick, IEEE Trans. Electron Dev., ED-26, p. 1742, 1979.

[18] L. D. Edmonds, IEEE Trans. Nucl. Sci., 43, p. 2346, 1996. 